\title{
Management of Breast Cancer by Vaccine: Fact or Fiction
}

DOI: $\underline{\text { http://dx.doi.org/10.5915/44-1-9055 }}$

Soumen Das ${ }^{1}$, MS, FUICC, FMAS; Retina Paul' ${ }^{2}$ MD(PGT); Utpal De ${ }^{3}$, MS; Mrityunjay Mukherjee, ${ }^{3}$ MS

${ }^{1}$ Institute of Post Graduate Medical Education \& Research and Seth Sukhlal Karnani Memorial Hospital

\author{
Kolkata, West Bengal, India
}

${ }^{2}$ D.Y. Patil Vidyapeeth Medical College, Pune, Maharashtra, India

${ }^{3}$ Medical College, Kolkata, West Bengal, India

\begin{abstract}
Breast cancer (BC) is the most common malignancy (75-80\%) among women. Options for management of $\mathrm{BC}$ are multivariate. Available modalities include surgery, radiotherapy, chemotherapy and hormone therapy. Despite availability of improved therapeutic adjuncts, mortality from BC is $40 \%$. Vaccination strategies against $\mathrm{BC}$ are emerging as a viable alternative. This review highlights the available results of this emerging therapeutic strategy.
\end{abstract}

Key words: Breast cancer, Vaccine

Breast cancer $(\mathrm{BC})$ is the most common malignancy (75-80\%) among women. ${ }^{1}$ Options for management of $\mathrm{BC}$ are multivariate. Available modalities include surgery, radiotherapy, chemotherapy and hormone therapy. Despite availability of improved therapeutic adjuncts, 39,970 BC deaths were expected in 2011. ${ }^{2}$

Vaccination strategies against $\mathrm{BC}$ are emerging as a viable alternative. Tumor immunology is one of the exploding fields in cancer research. Evidence of development of subtle degree of antitumor immunity by the host has been reported in literature 3 This has encouraged in-vitro formulation of active and passive immunization strategies against cancer. Malignancies like cervical cancer have specific infective agents as their principal etiology and vaccination against them is relatively easy. ${ }^{4}$ But in other cancers, like $\mathrm{BC}$, developing a vaccine depends on identifying

Correspondence should be directed to:

Soumen Das, MBBS,MS,FUICC,FMAS drsoumen_das@yahoo.co.in appropriate tumor antigen. Groups of antigens are expressed in BC, often more than one in a single patient. The initial strategy of developing a vaccine based on a single antigen has thus failed to achieve clinical significance. Later vaccines targeting multiple antigens were investigated. Many of these were combined with chemotherapy and hormone therapy to get the best possible result. This review highlights the available results of this emerging therapeutic strategy.

\section{Vaccination Strategies}

Vaccines targeting different tumor antigens have been developed for clinical use. Some have definite antigenic targets. These function by manipulating B-cell, T-cell or antigen presenting cell (APC) activity. Some vaccines act by directly activating humoral immunity. A second modality of immunotherapy is passive immunization with preformed antibodies against tumor antigens.

\section{Antigens}

$\mathrm{BC}$ patients develop subtle degree of immunity 
against certain antigens like MUC- 1 and HER2/neu. A variety of other antigens has been identified. These include Carcino Embryonic Antigen (CEA), p53, Sialyl -Tn (STn), melanomaassociated antigen (MAGE), GAGE, BAGE, XAGE and h TERT , alpha -lactalbumin. $45,5,7,8,9$

\section{Clinical Trials with BC Vaccines}

HER-2/neu related vaccines

HER-2/neu is one of the most extensively tested vaccine targets. Vaccines act by targeting HER-2/neu extracellular and intracellular domains. The first clinical data on HER-2/ neu vaccine was published in $1999^{10}$ where anti HER $2 /$ neu vaccine with was used in stage III, IV HER$2 /$ neu positive $\mathrm{BC}$ over six months. The aim was to detect $C D 4+$ response. Ninety-two percent of the patients completing six vaccinations developed HER-2/neu immunity to at least one peptide component of the vaccine as measured by peptide-specific T-cell proliferation in vitro. Additionally development of immunity against some epitopes of HER-2/neu which were not delivered by vaccine and HER-2/neu specific delayed hypersensitivity was observed which indicated the activated $\mathrm{T}$ cell mediated localization of site of antigen deposition. This immunity persisted for at least one year in $38 \%$ of patients.

Further HLA- A2 restricted HER-2-derived peptide p369-377 given with GM-CSF adjuvant to patients with metastatic breast resulted in development of new peptide-specific delayed type hypersensitivity (DTH) and antigen-specific CTLs capable of lysing HER-2/neu expressing tumors."

Vaccines to stimulate HER2 intracellular domain (ICD)-specific $\mathrm{T}$ cell and antibody were administered intradermally/subcutaneously four times at 3-week intervals to patients with stage II, III, or IV breast cancer with more than 50\% HER2 over-expressing tumor cells who were disease-free after surgery and adjuvant therapy. ${ }^{12}$ ICD-specific
$\mathrm{T}$ cell and antibody responses were measured. Delayed-type hypersensitivity (DTH) reactions at the injection site and HER2 specificity was detected by cytokine flow cytometry in majority of the patients. At more than 5 years of follow-up, $86 \%$ (six out of seven) had detectable anti-ICD antibodies. One patient experienced a pulmonary recurrence at 4 years. Although this was a small pilot study, the well-tolerated nature of the vaccines, the lack of cardiac toxicity, significant immunogenicity, and a 100\% 4.5-year survival rate suggest that vaccination with HER2 ICD is appropriate for further study..$^{13}$

In US Military Cancer Institute Clinical Trials Group Study I-01 and I-02 E75, a HER-2/neuderived peptide, was administered as a preventive vaccine with granulocyte-macrophage-colonystimulating factor (GM-CSF) in disease-free lymph node-positive and lymph node-negative $B C$ patients. The optimal biologic dose (OBD) was determined based on toxicity and immunologic response. Patients were vaccinated over 6 months (3, 4, or 6 times) with different doses of E75 plus GM-CSF. Immunologic response was measured by DTH and E75-specific CD8+ T-cells were quantified. Ninety-nine patients were vaccinated in 7 dose groups. Results revealed a trend toward an increase in the average postvaccine dimer, a significantly larger DTH response and a trend toward decreased recurrences for larger tumors, more positive lymph nodes and high grade tumors with a median follow-up time of one and a half years. ${ }^{9,14}$

Recently developed DNA vaccination is a viable alternative to peptide vaccination to induce potent anti-tumor CD8 $\mathrm{T}$ cell responses that provide effective therapeutic benefit. ${ }^{15}$

Studies to evaluate the early immunotherapeutic targeting of HER-2/neu in ductal carcinoma in situ DCIS revealed encouraging results. ${ }^{15}$ Preoperative HER-2/neu positive DCIS patients, vaccinations of dendritic 
cells pulsed with HER-2/neu HLA class I and II peptides were done at four weekly intervals. Sixty-three per cent of the patients showed markedly decreased HER-2/neu expression in surgical tumor specimens, often with measurable decreases in residual DCIS, suggesting an active process of "immunoediting" for HER-2/neuexpressing tumor cells following vaccination. Vaccination strategies may therefore have potential for both the prevention and the treatment of early breast cancer.

\section{MUC1 related vaccines}

MUC1 is another widely investigated antigen in BC .Peptide or carbohydrate based vaccines have been developed. MUC1 remains mostly in unglycosylated form in cancer, and thus the antigens on the cancer surface are different from normal cell. ${ }^{16}$ Therefore targeting MUC1 for cancer immunotherapy can exploit the difference between cancer and normal cells, eliminating the cancerous cells while leaving the normal mammary cells unharmed. Preclinical trials with MUC1 showed that MUC1 is a relatively poor immunogen in humans. Anti-MUC1 tumor immunity has been initiated by vaccination of mice with the recombinant bacillus CalmetteGuérin-based breast cancer vaccine that coexpresses variable-number tandem repeats of MUC1 and CD80. ${ }^{17}$ Experimental studies using two recombinant bacillus Calmette-Guérin ( $\mathrm{BCG}$ ) vaccines (rBCG-MVNTR4-CSF and rBCG-MVNTR8CSF) have shown encouraging results. ${ }^{18}$ Moreover, evaluation of $\mathrm{N}$-terminal region (2-147 amino acids) of MUC1 (MUC1-N) for dendritic cell (DC)based cancer immunotherapy has yielded good results. ${ }^{19} \mathrm{GM}$-CSF has been shown to increase the percentage and activity of antigen-presenting cells. ${ }^{20}$ Post-translational modifications like phosphrylation and glycosylation of important functional motifs of MUC1 play an important role in presenting MUC1 as a candidate for breast cancer vaccine. ${ }^{21}$ TG4010 vaccine, which incorporates the MUC1 antigen into a nonpropagative pox viral vector, and interleukin-2 incorporated TG4010 as an immune stimulus have been tested in $\mathrm{BC}$ with encouraging results. ${ }^{22}$

DNA plasmids encoding human MUC1 (pMUC1) and mouse interleukin-18 (pmuIL-18) has demonstrated effective results in preventing and treating pulmonary metastases in animal models. ${ }^{23}$

\section{CEA-related vaccines}

Immunotherapy with these CEA cancer vaccines may prove most effective in the adjuvant setting, where disease has been controlled or stabilized with conventional therapies. The development of CEA cancer vaccines involves many parameters, including the appropriate form of the vaccine, i.e., recombinant protein, peptides, vectors etc., the use of classical adjuvants and/or biological adjuvants such as cytokines, and the use of $\mathrm{T}$ cell costimulatory molecules. ${ }^{24}$

\section{Poly Vaccine}

Poly vaccine regimen consisting of the genes for CEA and MUC-1, along with co-stimulatory molecules (TRICOM; composed of B7.1, intercellular adhesion molecule 1, and lymphocyte function-associated antigen 3), is well tolerated and safe and has shown evidence of clinical activity. ${ }^{25}$ It is associated with both CD8 and CD4 immune responses and has a $2 \%$ low grade injection-site reaction.

\section{Alfa-Lactalbumin, the Novel Vaccine}

Jaini from Clevelend clinic has worked on this new antigen,_alpha-lactalbumin, as target vaccine autoantigen because it is a breast-specific differentiation protein expressed in high amounts in the majority of human breast carcinomas and in mammary epithelial cells only during lactation. This vaccine has experimentally proved to induce mammary gland failure during lactation and 
tumorogenesis, thus preventing breast cancer. But the problems of this vaccine are lactation failure and the fact that not all the $\mathrm{BC}$ patients express alfa-lactalbumin. ${ }^{26}$

\section{Discussion}

High mortality despite modern treatment modalities was the impetus for the search for alternative therapy. Immunotherapy came up as a viable modality. Passive immunization of $B C$ patients with Trastuzumab has been used clinically with moderate results. Active immunization with vaccines targeting different tumor antigens is one of the exploitable fields in biotechnology. Different preclinical and clinical studies have reported a variety of results as discussed previously, but none showed gold standard results. The prime difficulty encountered during vaccine development was the continuous dynamic interaction between tumor cell and host immunity. Each modified the other. Thus experimentally successful vaccines did not show promising result in clinical studies. There is downregulation of antigen when therapeutic antibody is used. This is known as "Antigen loss variant resistance. " ${ }^{25}$ This necessitates the formulation of vaccines targeting multiple antigens. Even these polyvalent vaccines developed resistance because of down-regulation of components of antigen processing pathway like MHC I/II, Transporter associated protein. ${ }^{25}$ Furthermore, all the tumor antigens are recognized as self antigen by host immunity. Studies demonstrated that there is development of immune suppression in patients with BC.

\section{Conclusion}

Vaccination is a novel modality in breast cancer treatment. Studies have shown mixed results. Moreover, issues related to use of vaccine for prevention or treatment, determination of dosage and suitability as a primary or adjuvant therapy are yet to be solved. Further studies are to be conducted to establish this as a prime therapy of $\mathrm{BC}$. Data on ongoing trials on $\mathrm{BC}$ vaccine are available on National Cancer Institute (NCI) website. ${ }^{26}$

\section{References}

1. Bray F, McCarron P, Parkin DM. The changing global patterns of female breast cancer incidence and mortality. Breast Cancer Res. 2004;6:229-39. http://dx.doi.org/10.1186/bcr932

2. Chen YT, Güre AO, Tsang S, et al. Identification of multiple cancer/testis antigens by allogeneic antibody screening of a melanoma cell line library. Proc Natl Acad Sci U S A. 1998;95:6919-23. http://dx.doi.org/10.1073/pnas.95.12.6919 3. Gravitt PE, Shan KV. A virus-based vaccine may prevent cervical cancer. Curr Infect Dis Rep. 2005;7:125-31. http://dx.doi.org/10.1007/s11908005-0072-3

4. Scanlan MJ, Gout I, Gordon CM, et al. Humoral immunity to human breast cancer: antigen definition and quantitative analysis of mRNA expression. Cancer Immun. 2001;1:4. PMID: $\underline{12747765}$

5. Runnebaum IB, Nagarajan M, Bowman M, et al. Mutations in p53 as potential molecular markers for human breast cancer. Proc Natl Acad Sci U S A. 1991;88:10657-61.

http://dx.doi.org/10.1073/pnas.88.23.10657

6. Singh R, Paterson Y. Vaccination strategy determines the emergence and dominance of CD8+ $\mathrm{T}$-cell epitopes in a FVB/N rat HER-2/neu mouse model of breast cancer. Cancer Res. 2006;66:774857. http://dx.doi.org/10.1158/0008-5472.CAN-05$\underline{4469}$

7. Murray JL, Gillogly ME, Przepiorka D, et al. Toxicity, immunogenicity and induction of E75specific tumor-lytic CTLs by HER-2 peptide E75 (369-377) combined with granulocyte macrophage colony stimulating factor in HLA-A2+ patients with metastatic breast and ovarian cancer. Clin Cancer 
Res. 2002;8:3407-18. PMID: 12429628

http://clincancerres.aacrjournals.org/content/8/1 1/3407.short

8. Wei H, Wang S, Zhang D, et al. Targeted delivery of tumor antigens to activated dendritic cells via CD11c molecules induces potent antitumor immunity in mice. Clin Cancer Res. 2009;15:4612-21. http://dx.doi.org/10.1158/1078-0432.CCR-08-3321

9. Cho HI, Niu G, Bradley N, et al. Optimized DNA vaccines to specifically induce therapeutic CD8 T cell responses against autochthonous breast tumors. Cancer Immunol Immunother. 2008;57:1695-703.

http://dx.doi.org/10.1007/s00262-008-0465-X 10. Czerniecki BJ, Koski GK, Koldovsky U, et al. Targeting HER-2/neu in early breast cancer development using dendritic cells with staged interleukin-12 burst secretion. Cancer Res. 2007;67:1842-52. http://dx.doi.org/10.1158/00085472.CAN-06-4038

11. Ohyabu N, Hinou H, Matsushita T, et al. An essential epitope of anti-MUC1 monoclonal antibody KL-6 revealed by focused glycopeptide library. J Am Chem Soc. 2009;131:17102-9. http://dx.doi.org/10.1021/ja903361f

12. Yang E, Hu XF, Xing PX. Advances of MUC1 as a target for breast cancer immunotherapy. Histol Histopathol. 2007;22:905-22. PMID: 17503348 13. Yuan S, Shi C, Lv Y, et al. A novel Bacillus Calmette-Guérin-based breast cancer vaccine that coexpresses multiple tandem repeats of MUC1 and CD80 breaks the immune tolerance and inhibits MUC1-positive breast cancer growth. Cancer Biother Radiopharm. 2009;24:607-13.

http://dx.doi.org/10.1089/cbr.2009.0622

14. Yuan S, Shi C, Ling R, et al. Immunization with two recombinant Bacillus Calmette-Guérin vaccines that combine the expression of multiple tandem repeats of mucin- 1 and colony stimulating-factor suppress breast tumor growth in mice. J Cancer Res Clin Oncol. 2010;136:1359-67.

http://dx.doi.org/10.1007/s00432-010-0787-x
15.Yang H, Cho NH, Seong SY. The Tat-conjugated $\mathrm{N}$-terminal region of mucin antigen 1 (MUC1) induces protective immunity against MUC1expressing tumours. Clin Exp Immunol. 2009;158:174-85. http://dx.doi.org/10.1111/j.13652249.2009.03997.x

16.Yuan S, Shi C, Liu L, et al. MUC1-based recombinant Bacillus Calmette-Guerin vaccines as candidates for breast cancer immunotherapy. Expert Opin Biol Ther. 2010;10:1037-48. http://dx.doi.org/10.1517/14712598.2010.485185 17. Begum M, Khurshid R, Saleem A, et al. MUC1 based breast cancer vaccines: role of post translational modifications. J Ayub Med Coll. Abbottabad. 2008;20:130-3. PMID: 19999225 18. Acres B. Cancer immunotherapy: phase II clinical studies with TG4010 (MVA-MUC1-IL2). J BUON. 2007;12 Suppl 1:S71-5. PMID: 17935281 19. Hodge JW. Carcinoembryonic antigen as a target for cancer vaccines. Cancer Immunol Immunother. 1996;43:127-34. http://dx.doi.org/10.1007/s002620050313 20.Gulley JL, Arlen PM, Tsang KY, et al. Pilot study of vaccination with recombinant CEA-MUC-1TRICOM poxviral-based vaccines in patients with metastatic carcinoma. Clin Cancer Res.

2008;14:3060-9. http://dx.doi.org/10.1158/10780432.CCR-08-0126

21.Holmberg L, Oparin DV, Gooley T, et al. The role of cancer vaccines following autologous stem cell rescue in breast and ovarian cancer patients: experience with the STn-KLH vaccine (Theratope). Clin Breast Cancer. 2003;3 Suppl 4:S144-51. http://dx.doi.org/10.3816/CBC.2003.s.004 22.Knutson KL, Schiffman K, Disis ML. Immunization with a HER-2/neu helper peptide vaccine generates HER-2/neu CD8 T-cell immunity in cancer patients. J Clin Invest. 2001;107:477-84. http://dx.doi.org/10.1172/JCI11752 23.Marincola FM, Jaffee EM, Hicklin DJ, et al. Escape of human solid tumors from T-cell recognition: molecular mechanisms and 
functional significance. Adv Immunol. 2000;74:181-273. http://dx.doi.org/10.1016/S00652776(08)60911-6

24.Walker LS, Abbas AK. The enemy within: keeping self-reactive $\mathrm{T}$ cells at bay in the periphery. Nat Rev Immunol. 2002;2:11-9. http://dx.doi.org/10.1038/nri701 25.Jaini R, Kesaraju P, Johnson JM, et al. An autoimmune-mediated strategy for prophylactic breast cancer vaccination. Nat Med. 2010;16:799-
803. http://dx.doi.org/10.1038/nm.2161

26. National Cancer Institute at the National Institutes of Health. [Stored protocol search: Cancer Type/Condition: Breast cancer, female | Trial Type: Treatment | Treatment/Intervention: vaccine therapy | Trial Status: Active] http://www.cancer.gov/clinicaltrials/search/resu lts?protocolsearchid=6371711 [Accessed 2013-Apr22] 